\title{
Simulation of Optical Distortions of Two-Dimensional Shear Layer Using Large Structure Model (LSM)
}

\author{
Stanislav Gordeyev ${ }^{*}$, Eric J. Jumper ${ }^{\dagger}$ \\ University of Notre Dame, Notre Dame, IN, 46556 \\ and \\ Jim Trolinger ${ }^{\ddagger}$ \\ MetroLaser Incorporated, Irvine, CA, 92614
}

\begin{abstract}
A simple weakly compressible discrete vortex Large Structure Model (LSM) to predict dynamic evolution of the subsonic compressible two-dimensional shear layer is presented. It was shown that LSM code correctly predicts the shear layer growth rate and structure evolution, as well as spectral content and amplitude of optical distortions imposed by the shear layer on the passing laser beam. Due to small number of vortices required to represent the shear layer dynamics, computational times are very short. Also, the model was successfully extended to predict optical aberrations in flows over rectangular cavities. This model was developed as an engineering tool to quickly estimate dynamical optical distortions from shear layers.
\end{abstract}

\section{Introduction}

The presence of large-scale vortical structures in a compressible transonic shear layer is well established and has been thoroughly investigated, see, for instance Ref. 1 and references therein. These vortical structures are shown to create low-pressure drops to balance the centrifugal forces and, consequently create regions of lower density and index of refraction inside of the structures ${ }^{2}$. From an aero-optical point of view, these high-frequency aberrations are very destructive to a laser beam passing through the shear layer, leading to dispersing of the laser beam energy away from a target ${ }^{3,4}$. These structures impose a great challenge on the adaptive optics corrections because of their high levels of optical aberrations and corresponding high frequency bandwidths (Ref. 5 and references therein).

It is well known that the main mechanism for creating vortical structures in a shear layer is a Kelvin-Helmholtz inviscid, inflectional instability of the mean velocity profile for a fairly broad range of frequencies ${ }^{1,6}$. The main dynamical mechanism to develop vortical structures is as following ${ }^{7}$ : All of these unstable frequencies go through an exponential growth, although with different growth rates. The harmonic with the highest growth rate, called a fundamental harmonic eventually outgrows all other harmonics and leads to an appearance of

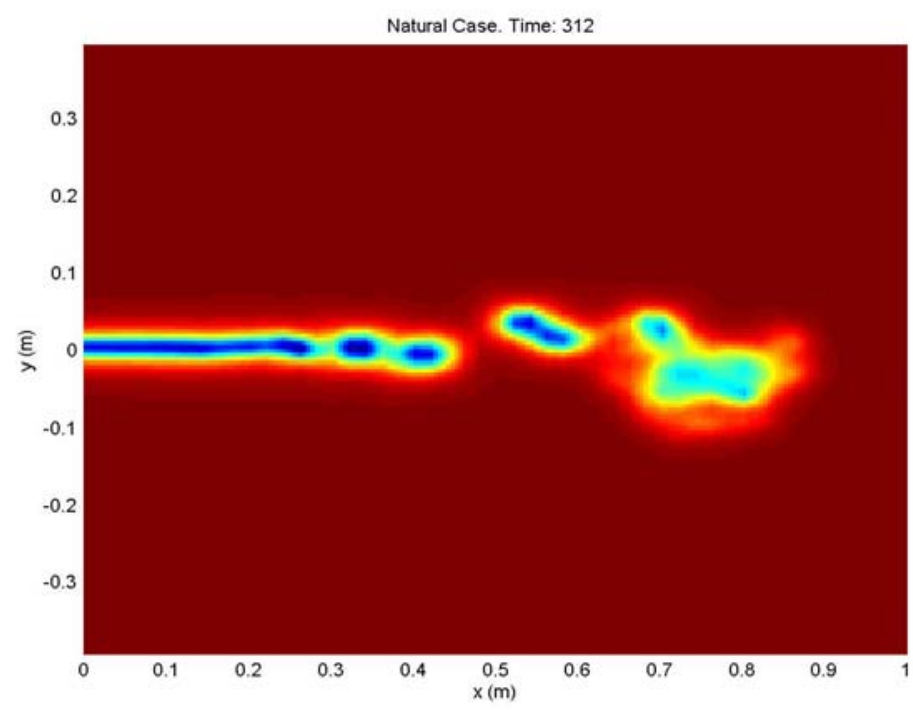

Figure 1. Vorticity field for 2-D shear layer computed using DVM method.

\footnotetext{
* Research Assistant Professor, Department of Aerospace and Mech. Eng., Member AIAA.

${ }^{\dagger}$ Professor, Department of Aerospace and Mech. Eng., Fellow AIAA.

‡ Vice President \& Director of Research, MetroLaser Incorporated, Member AIAA.
} 
two-dimensional spanwise vortical structures in the shear layer some distance $\mathrm{X}_{\text {roll }}$ downstream, $\mathrm{x}_{\mathrm{roll}} \sim 3 \Lambda=3 \mathrm{U}_{\mathrm{c}} / \mathrm{F}$. Here $\mathrm{U}_{\mathrm{c}}$ is the shear layer convective speed, $\Lambda$ is the fundamental wavelength and $\mathrm{F}$ is the fundamental frequency; the latter is related to the initial shear layer momentum thickness, $\theta_{0}$, as $\theta_{0} \mathrm{~F} / \mathrm{U}_{\mathrm{c}}=0.032$. Structures go through a series of pairing scenarios further downstream due to the Biot-Savart interaction, when two or more neighboring structures merge and a stronger vortical structure appears as a result. These structures continue to pair and merge downstream, thus the shear layer growth increases downstream. An example of a vorticity field in a 2-D transonic shear layer computed using a Weakly Compressible Discrete Vortex Model (DVM) ${ }^{2,5}$, is given in Figure 1. In this example, the vortical structure appears around $0.2 \mathrm{~m}$ and the first and the second pairing can be observed at $\mathrm{x}=0.53$ and $0.74 \mathrm{~m}$, consequently. Some average quantities predicted by DVM code are presented in Figure 2. Of course, three-dimensional effects will eventually break down the spanwise vortical structure, which further complicates this already rich 2-D shear layer dynamics.

To re-iterate, the 2-D shear layer exhibits the following dynamical features:

(a) A compact vortical structure appears some distance downstream from the beginning of the shear layer.

(b) These structures go through subsequent series of pairing and merging events further downstream.

(c) An average structure size grows linearly downstream because of sequence of pairing events.

Direct numerical or any other large-eddy-based simulations of the turbulent shear layer for high Reynolds numbers and transonic Mach numbers require an significant amount of computational resources. Even the inherently 2-D,inviscid $\mathrm{DVM}^{2,5}$ uses many thousands of vortices to model the dynamics of the shear layer and it still takes hours to compute the shear layer dynamics on a regular PC. Therefore, there is a need to create a very simple structure-based semi-empirical shear layer model which captures essential shear layer features and allows to correctly predict statistical properties of the shear layer, like mean velocity and turbulent profiles and a growth rate and to create instantaneous velocity and related density field realizations, yet can be run quickly on any computer. Furthermore, the model should simulate realistic time series of different pairing events in the shear layer. This model could be a very useful engineering tool when someone needs to quickly estimate shear layer scales and spatialtemporal characteristics of optical aberrations imposed on a laser beam while designing an adaptive optics control strategy, for instance.
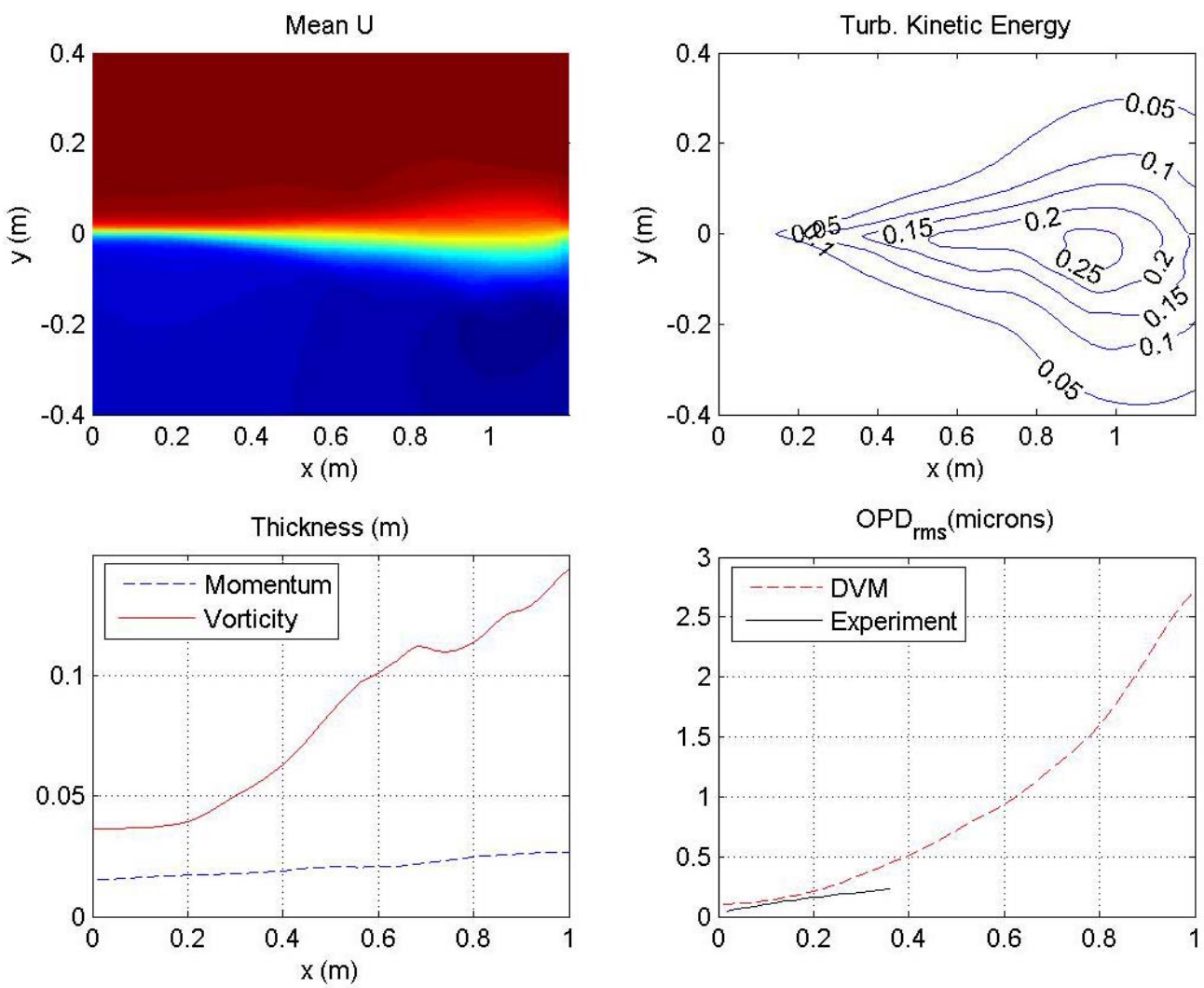

Figure 2. Average quantities and OPD variation predicted by DVM code. 


\section{Large-Structure Model}

The Discrete Vortex approach ${ }^{8,9}$ is a Langangian-based, grid-free model which approximates a shear layer as a number of free-moving solid core vortices which interact with each other via the Biot-Savart induction. For details in DV basics and further developments the reader is referred to Refs. 2, 8, 9 and 10. DV approach correctly predicts all essential, both mean and instantaneous, features of the two-dimensional shear layers like the shear layer growth rate, velocity profiles, turbulence intensity, structure size and dynamics. It makes DV modeling an attractive code for predicting shear layer dynamics.

The presented model is a further simplification of DV approach. Instead of representing shear layer structures with a large number of small vortices (which does allow predicting the correct structure shape and the paring mechanism), a simple Large Structure Model (LSM) models each vortical structure as a single round vortex with a fixed size. It allows to drastically dropping number of vortices in the computational domain to represent the shear layer dynamics, thus significantly decreasing computational times to run the code, while still resolving the essential behavior of the shear layer.

Referring to the shear layer dynamics discussion presented above, LSM models the shear layer as a small number of round vortices appearing in quasi-periodic fashion with an initial circulation $\Gamma_{0}=$ (Uupper-Ulower) $\Lambda$ and size $\mathrm{a}_{0}=0.3 \Lambda$ at the roll-up location $\mathrm{x}_{\text {roll }}$ and let them freely convect downstream and interact with each other. Uupper and Ulower are the high and the low speeds on both sides of the shear layer. Since the structure shape is now fixed, the vortex pairing and merging is parametrically modeled via a simplified mechanism outlined below. The mean velocity field $\mathrm{U}(\mathrm{x}, \mathrm{y})$ is one of the input parameters and should be provided from either experiment or numerical calculations.

The following simplifications and assumptions were used in this model:

1. Each vortex is located at (xj, yj) and has a finite round core of radius aj and a strength $\Gamma \mathrm{j}$ (Figure 3 ) with an exponential vorticity distribution $\omega_{j}(r)=\Gamma_{j} /\left(2 \pi a_{j}^{2}\right) \exp \left(-1.256 r^{2} / a_{j}^{2}\right)$; the azimuthal velocity profile defined as

$$
u_{\theta}^{j}(r)=\frac{\Gamma_{j}}{2 \pi r}\left[1-\exp \left(-1.256 \frac{r^{2}}{a_{j}^{2}}\right)\right],\left.\quad \frac{d u_{\theta}^{j}(r)}{d r}\right|_{r=a}=0
$$

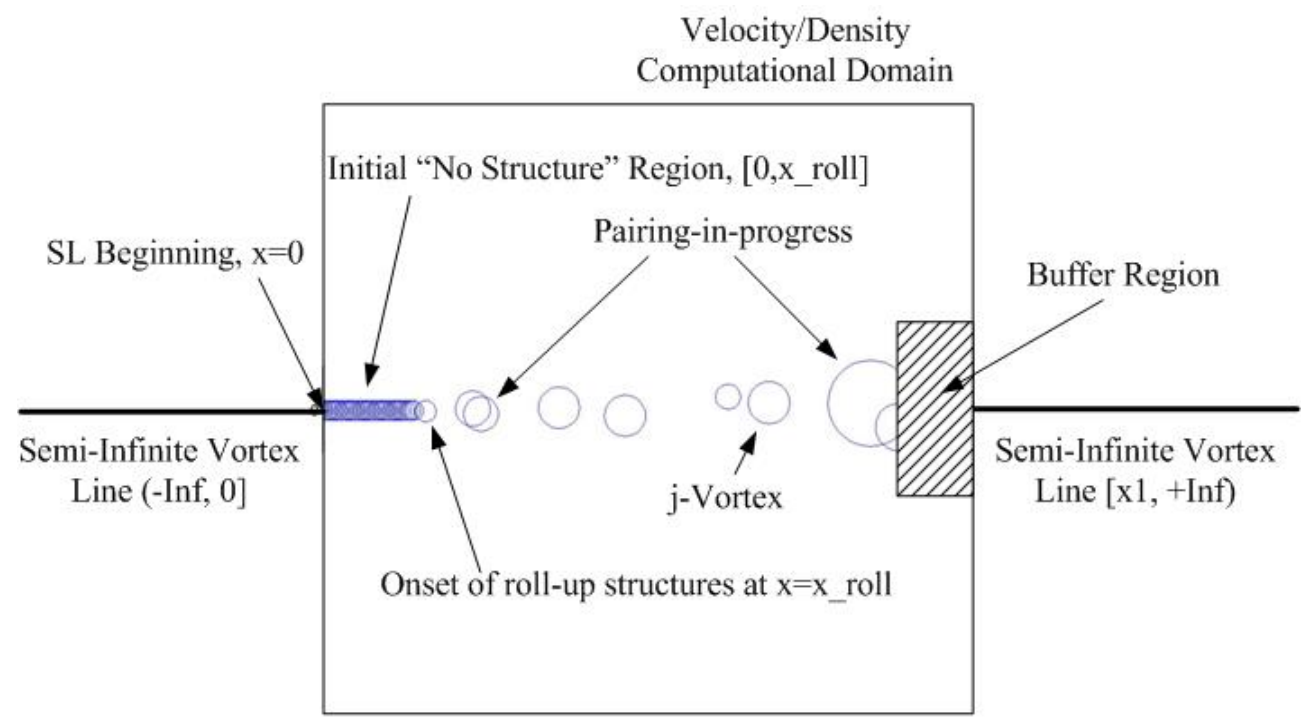

Figure 3. Large Structure Model (LSM).

2. To simplify the vortex interaction process, each vortex interacts "directly" only with 2 neighboring vortices up- and downstream via Biot-Savart induction and the rest of the shear layer "indirectly" via the provided mean velocity profile, 


$$
\begin{aligned}
& \frac{d x_{j}}{d t}=u_{j}=\alpha \sum_{i=j-1, j+1} u_{\theta}^{i}\left(r_{i j}\right) \frac{\left(y_{j}-y_{i}\right)}{r_{i j}}+U(x, y) \\
& \frac{d y_{j}}{d t}=v_{j}=-\alpha \sum_{i=j-1, j+1} u_{\theta}^{i}\left(r_{i j}\right) \frac{\left(x_{j}-x_{i}\right)}{r_{i j}}, r_{i j}^{2}=\left(x_{j}-x_{i}\right)^{2}+\left(y_{j}-y_{i}\right)^{2}
\end{aligned}
$$

The $\alpha$-parameter is introduced to adjust the pairing speed and therefore to control the shear layer growth rate.

3. To model the pairing and merging process, the vortex size grows depending on the $\mathrm{x}$-distance to the closest vortex (labeled near),

$$
a_{j}=\tilde{a}_{j}+\tilde{a}_{\text {near }} \exp \left[-\left(x_{j}-x_{\text {near }}\right)^{2} /\left(\tilde{a}_{j}+\tilde{a}_{\text {near }}\right)^{2}\right]
$$

where the tilde denotes the original vortex size before the pairing.

4. Merging happens when the x-distance between vortices becomes less than 0.1 of the vortex radius and merging vortices are replaced with one vortex with the combined strength of participating vortices and proper radius placed at the "center-of-mass".

5. The initial "no-structure" region (Figure 3) is represented as a number of fixed vortices with proper strengths to represent the initial shear layer thickness.

6. The flow up- and downstream of the computational region is modeled as two-semi-infinite vortex sheets with proper strength to match velocity boundary values at $y=+/-\infty$, see Figure 3 .

7. To eliminate the "jerkiness" in the velocity field when the vortex leaves the computational region, the buffer region is introduced between $\mathrm{x}$ _buffer and x1, see Figure 3, to smoothly blend the outgoing vortex into the semi-infinite vortical sheet.

8. The new vortex is inserted at the $x_{\text {roll }}+N(0, s)$ location every $T=1 / F$ period, where $F$ is the fundamental shear layer frequency. The Gaussian noise with zero mean and s-deviation $\mathrm{N}(0, \mathrm{~s})$ is introduced to model some degree of randomness in the structure's initial position.

9. The system (2) is numerically solved using $1^{\text {st }}$ order Euler method with $\Delta \mathrm{t}=0.1 \mathrm{~T}$ and the velocity field is calculated inside of the computational domain at every time step,

$$
\begin{aligned}
& u(x, y, t)=\sum_{i}^{N} u_{\theta}^{i}\left(r_{i j}\right) \frac{\left(y-y_{i}\right)}{r_{i j}}+U_{\text {semi-inf }} \\
& v(x, y, t)=\sum_{i}^{N} u_{\theta}^{i}\left(r_{i j}\right) \frac{-\left(x-x_{i}\right)}{r_{i j}}+V_{\text {semi-inf }}
\end{aligned}
$$

where $(\mathrm{U}, \mathrm{V})_{\text {semi-inf }}$ is velocity field from both upstream and downstream semi-infinite vortex sheets.

LSM has the following input parameters:

Uupper, Ulower - velocities above and below the shear layer.

$\mathrm{U}(\mathrm{x}, \mathrm{y})$ - the mean velocity profile.

$\mathrm{F}$ - the fundamental frequency based on the initial momentum thickness.

$\Lambda=\mathrm{Uc} / \mathrm{F}$ - initial structure spacing based on the fundamental frequency the mean convective speed $\mathrm{Uc}=0.5 *$ (Upper+Ulower).

$\Gamma_{0}, \mathrm{a}_{0}-$ the initial structure circulation and size.

$\mathrm{x}_{\text {roll }}$ - location of the first roll-up.

$\mathrm{x} \_$buffer - beginning of the buffer region.

xend - the end $f$ the computational domain.

$\mathrm{x} 1$ - beginning of the semi-infinite vortex sheet extending to + infinity.

$\alpha$ - parameter responsible for the shear layer growth rate.

$\mathrm{s}$ - level of randomness in the initial structure spacing.

Knowing the velocity field at any given time, the pressure $p$ and the temperature $T$ fields can be estimated, using an adiabatic approximation ${ }^{2}$, 


$$
\begin{aligned}
& \nabla^{2} p=-2 \rho\left(\frac{\partial u}{\partial x} \frac{\partial v}{\partial y}+\frac{\partial v}{\partial x} \frac{\partial u}{\partial y}\right)+\text { proper B.C. } \\
& T / T_{a d}=\left(p / p_{0}\right)^{(\gamma-1) / \gamma}, \quad \text { where } T_{a d}=T_{0}-\frac{\gamma-1}{2 \gamma R}\left(u^{2}+v^{2}\right)
\end{aligned}
$$

Here $\mathrm{p}_{0}$ and $\mathrm{T}_{0}$ are total pressure and temperature values. The density field is related to pressure and temperature fields via the equation of state $p=\rho R T$.

Note that the pressure equation in (5) needs the density field $\rho$ to be resolved. Thus, an iterative procedure was used to solve these equations:

1. An initial uniform pressure field was assumed, $\mathrm{p}^{(0)}(\mathrm{x}, \mathrm{y})=\mathrm{p}_{0}$.

2. Initial temperature and density fields were calculated, $\mathrm{T}^{(0)}(\mathrm{x}, \mathrm{y})$ and $\rho^{(0)}(\mathrm{x}, \mathrm{y})$.

3. The initial density field $\rho^{(0)}$ was used to compute a next approximation of the pressure field $\mathrm{p}^{(1)}$.

4. Steps 2 and 3 were repeated several times until the converged solution is found.

Knowing the density field, Optical Path Differences, OPDs are calculated using the Gladstone-Dale constant by integrating along lines normal to the shear layer,

$$
O P L(x, t)=K_{G D} \int_{-\infty}^{+\infty} \rho(x, y, t) d y, O P D(x, t)=O P L(x, t)-O P L(x=0, t) .
$$

Note that $O P D$ is defined slightly differently to satisfy $O P D(x=0, t)=0$.

\section{Results}

\section{A. Free Shear Layer}

In order to compare LSM code with the DVM code ${ }^{5}$, and the experimental data ${ }^{11,12}$, the LSM-code was used with following input parameters: Uupper $=261.43 \mathrm{~m} / \mathrm{sec}$, Ulower $=34.7 \mathrm{~m} / \mathrm{sec}, \Lambda=0.06 \mathrm{~m}, \mathrm{x}_{\text {roll }}=0.17 \mathrm{~m}$, x_buffer=0.8 m, xend $=1.1 \mathrm{~m}, \mathrm{x} 1=1.2 \mathrm{~m}, \mathrm{a}=0.3 \Lambda, \alpha=0.7, \mathrm{~s}=5 \% \Lambda, \Delta \mathrm{t}=0.1 / \mathrm{F}$. These parameters correspond to the shear layer with the initial momentum thickness of $\theta_{0}=0.002 \mathrm{~m}$ and the fundamental frequency of $\mathrm{F}=2466 \mathrm{~Hz}$. Velocity/density computational domain is $[0 \mathrm{~m} . .1 .1 \mathrm{~m}] \mathrm{x}[-0.4 \mathrm{~m} . .0 .4 \mathrm{~m}]$. The shear layer was calculated for 2000 time steps.

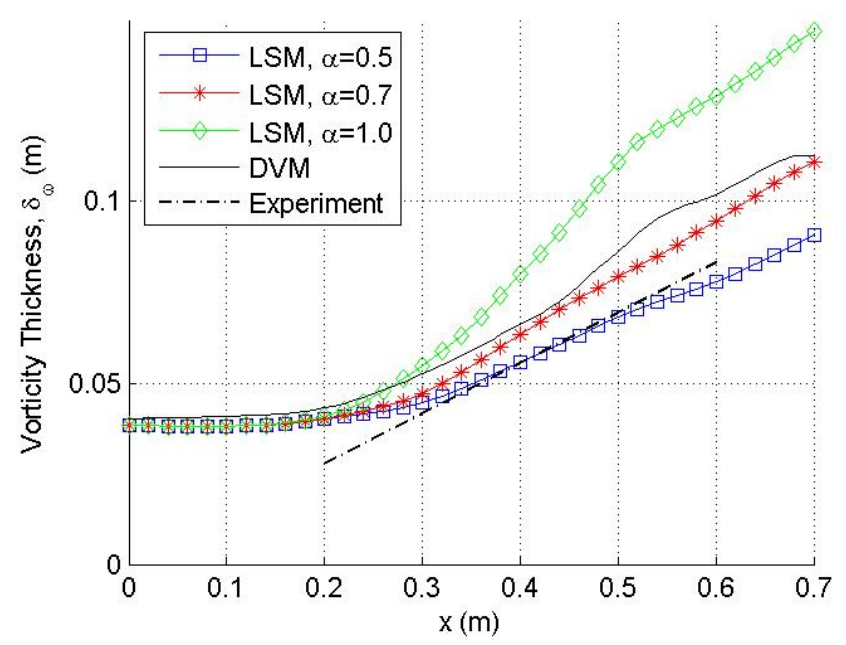

Figure 4. Vorticity thicknesses with different a-parameters.

Since vortex interactions were greatly simplified in the system (2), the $\alpha$-parameter must be tuned to match experimental data. The code predictions for the shear layer growth with different $\alpha$-parameters are presented in Figure 4. The vorticity thickness, $\delta_{\omega}$, is chosen to represent the local shear layer thickness, $\delta_{\omega}=\left(u_{\text {upper }}-u_{\text {lower }}\right) /\left.(d U / d y)\right|_{\max }$. When $\alpha$-parameter is too high, pairings occur faster, which force the shear layer to grow faster downstream. Similarly, small value of the $\alpha$-parameter will slow the pairing process, thus 
causing the shear layer to expand slower downstream. It was found that $\alpha=0.7$ provides the correct shear layer growth of $\mathrm{d} \delta_{\omega} / \mathrm{dx}=0.138$, observed in experiments ${ }^{13}$, as well as predicted by the DVM-code ${ }^{5}$.
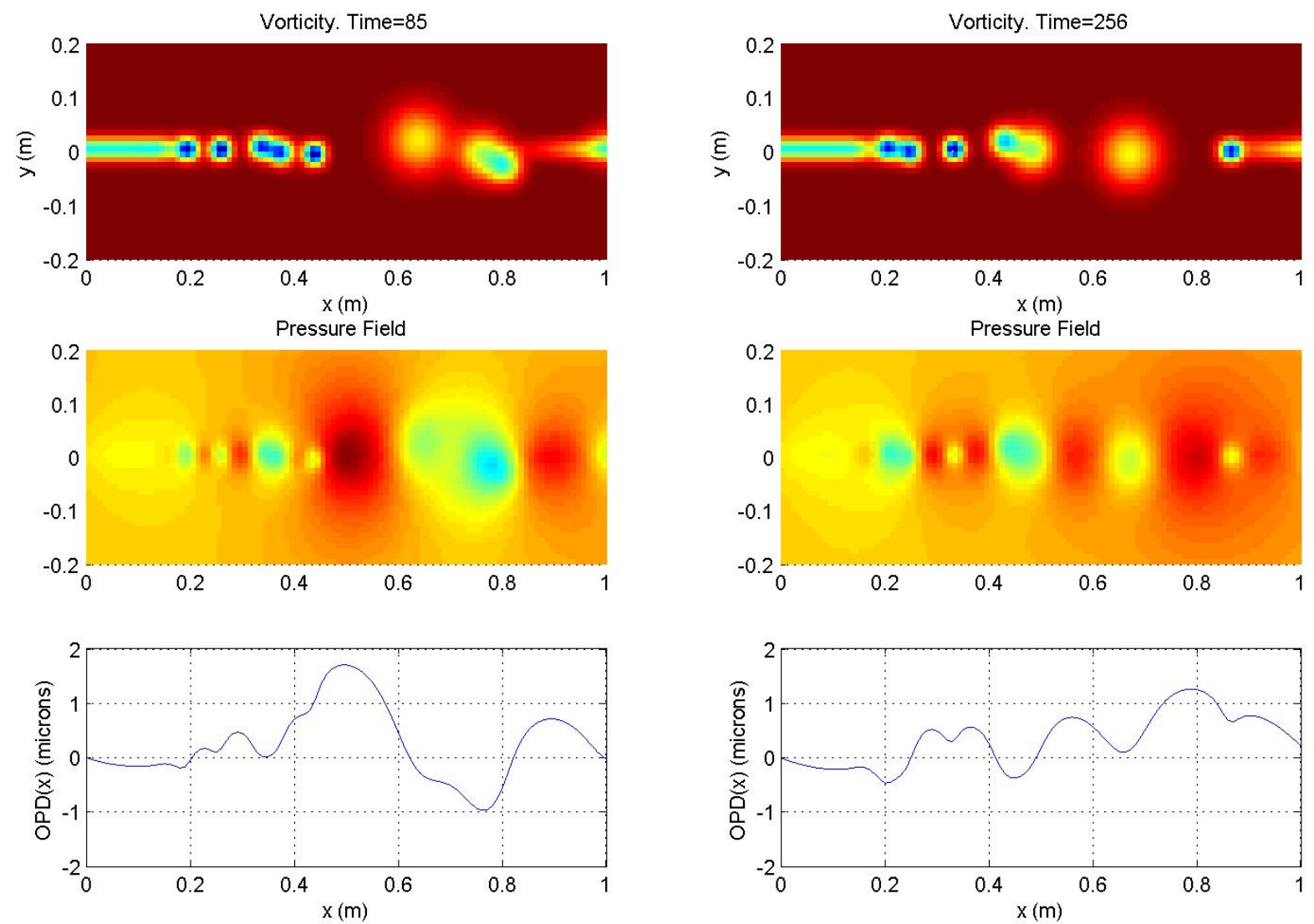

Figure 5. Two realizations of Vorticity and pressure fields and $\operatorname{OPD}(\mathrm{x}, \mathrm{t})$ from LSM code.

Once the proper value of the $\alpha$-parameter was found, the code was run for 2000 time steps and velocity, vorticity, pressure and OPDs were calculated at every time step as it was described before. Figure 5 presents two output realizations from LSM-code based on the mean velocity field, computed using DVM simulations ${ }^{5}$. The initial "no structure" region is present up to approximately 0.2 meters; by the end of this region round vortices are allowed to convect freely downstream and interact with each other as it was described before. Pairing processes can be clearly observed in these representative vorticity fields. Pressure fields have well defined drops or "wells" inside of vortices (marked as blue regions in Figure 5) as a result of balancing centrifugal forces inside vortical structures ${ }^{2}$. Also, it is interesting to note pressure peaks in between structures (red regions in Figure 5), for instance at $\mathrm{x}=0.5 \mathrm{~m}$ in the left plot in Figure 5. They represent stagnations regions between structures, where mutual effects of neighboring vortices cancel each other and the flow moves with the convective speed $U_{c}$. Consequently, OPDs are lower when integrated through vortical structures and higher when integrated in between structures; see OPD results in Figure 5.

Several average quantities: mean $U$, turbulent kinetic energy, shear layer vorticity thickness and temporal variation of OPD, OPD ${ }_{\text {rms }}$, are presented in Figure 6. Comparisons with DVM code ${ }^{5}$ and experimental data ${ }^{11}$ are also presented. From the vorticity thickness plot (Figure 6, the lower left plot) one can see that the LSM code, after a proper tuning predicts the correct initial thickness and the proper growth rate, as well as pairing and merging processes. Since LSM code resolves large structures only, it underpredicts the total turbulent kinetic energy, compared with DVM predictions (upper right plots in Figures 2 and 6). Comparison with experimental data ${ }^{11}$ shows the correct predictions in streamwise variations of $\mathrm{OPD}_{\mathrm{rms}}$ both in amplitude and trend, see lower right plot in Figure 6 . Note that the presence of the buffer region beyond $\mathrm{x} \_$buffer $=0.8 \mathrm{~m}$ causes all quantities to decrease, since vortex strengths in the buffer region were artificially decreased to be blended into the downstream semi-infinite vortical sheet at $\mathrm{x} 1=1.2 \mathrm{~m}$. 

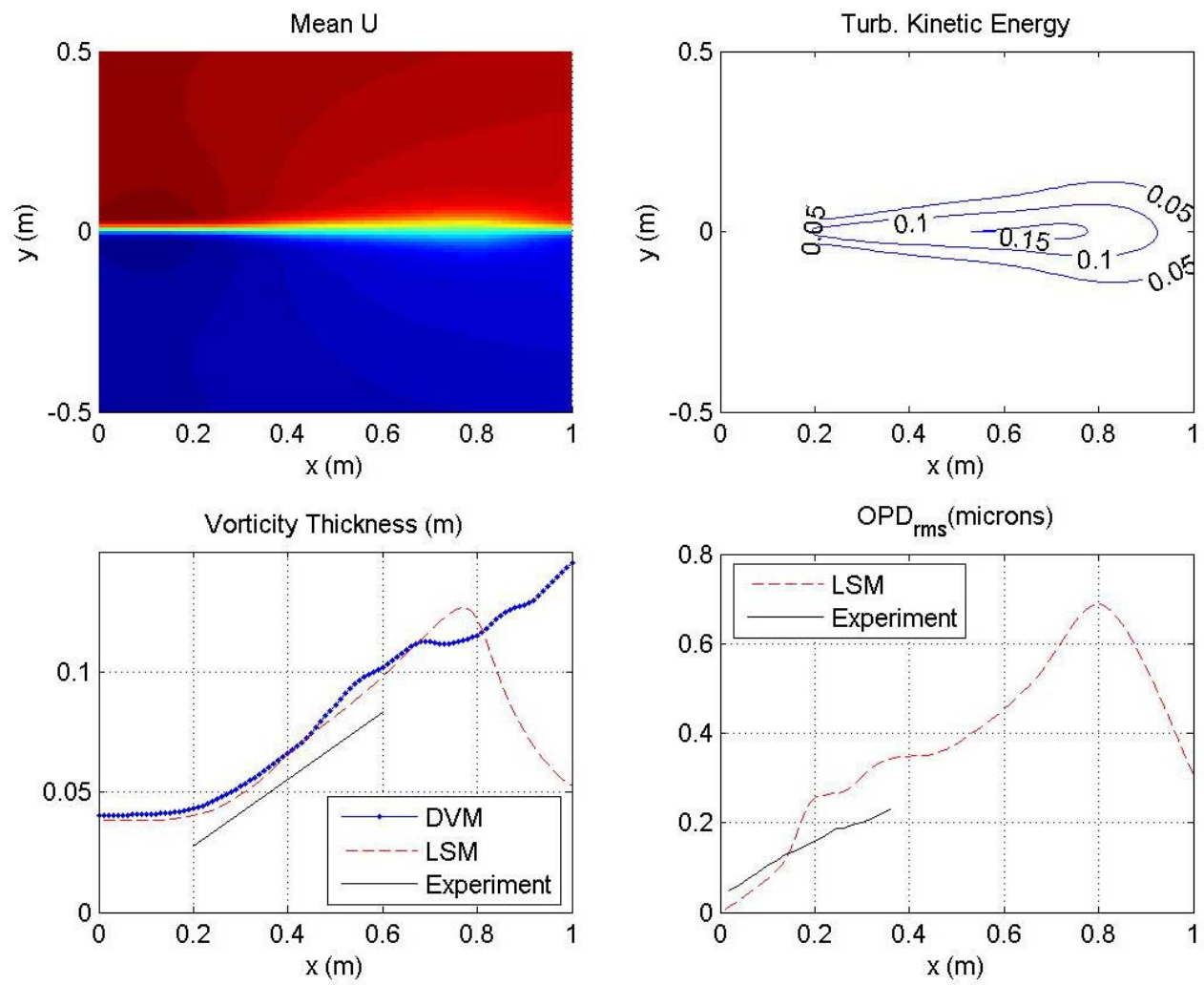

Figure 6. Average quantities predicted by LSM code.

Finally, temporal power spectral densities were computed from $\operatorname{OPD}(\mathrm{x}, \mathrm{t})$ and compared with both DVM-code and experimental results. LSM-code correctly predicts the streamwise evolution of spectra, both in shape and the location of the main peak. The narrow peak at $2.5 \mathrm{kHz}$ at $\mathrm{x}=0.2 \mathrm{~m}$ corresponds to the fundamental frequency $\mathrm{F}$. Thicker tails in experimental data, middle plot in Figure 7, are thought to be attributed to the presence of boundary layers on the tunnel walls ${ }^{11}$.
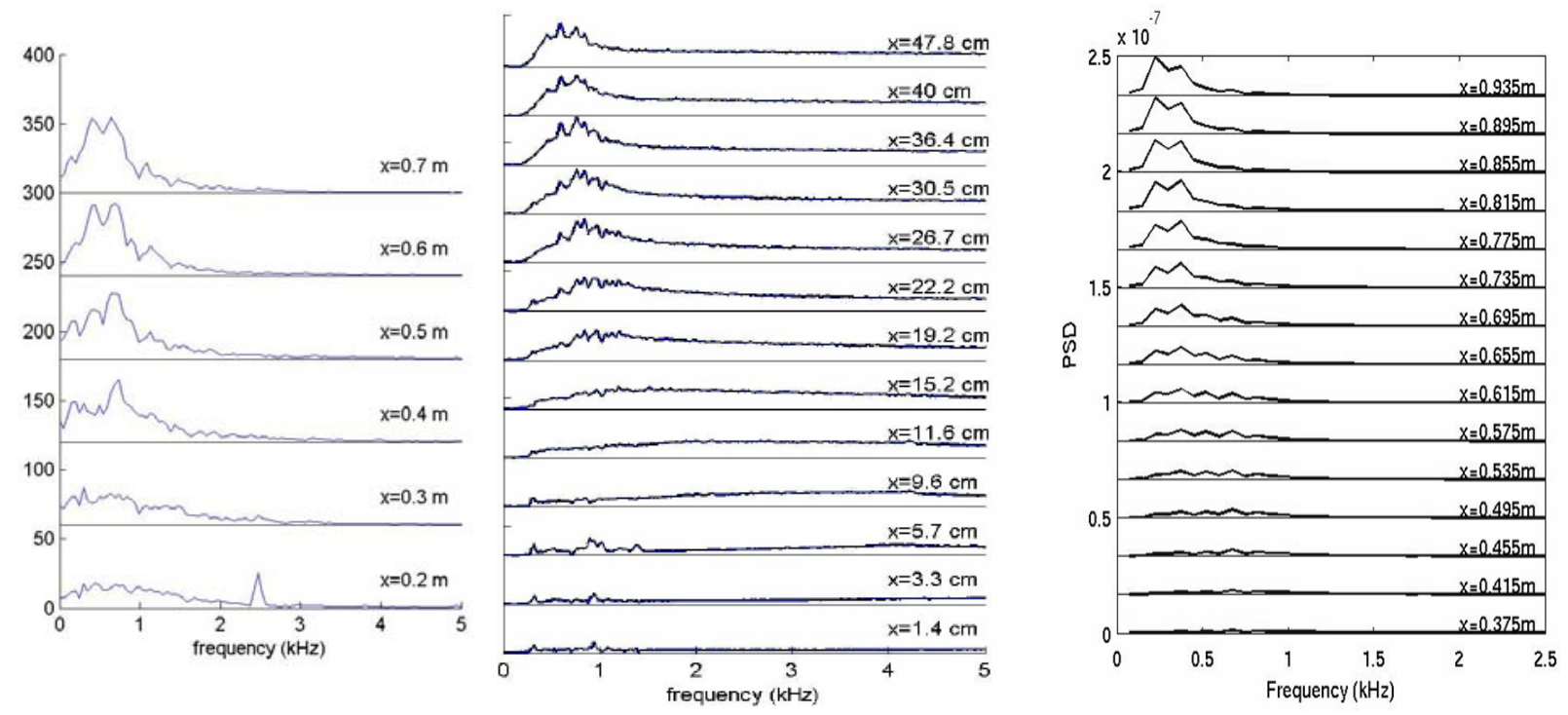

Figure 7. Streamwise evolution of OPD spectra: LSM-code (left plot), experimental results from Ref. 11 (middle plot) and DVM-code from Ref. 5 (right plot). 


\section{B. Cavity Flow}

The shear layer is also present over a rectangular cavity, but its evolution is modified by a presence of a strong feedback ${ }^{14-16}$ as follows: the shear layer develops large-structures through the inflection velocity profile instability; they convect downstream and impinge on the back wall of the cavity. It creates strong acoustical waves which travel upstream inside the cavity and impose disturbances on the initial region of the shear layer, thus completing the feedback. This mechanism leads to a creation of Rossiter modes ${ }^{17}$ in the cavity with modal frequencies $f_{n}$ given as ${ }^{18}$,

$$
S t_{n}=\frac{f_{n} L}{U_{\infty}}=\frac{n-\beta}{M_{\infty}\left(1+(\gamma-1) / 2 M_{\infty}^{2}\right)^{-1 / 2}+1 / k}
$$

where $n$ is the modal number, $L$ is the cavity length, $U_{\infty}$ and $M_{\infty}$ are freestream speed and Mach number, respectively, $k$ is the ratio between the shear layer convective speed and the freestream speed ( $k$ is typically 0.5..0.6) and $\beta=0.25$ is an empirical constant.

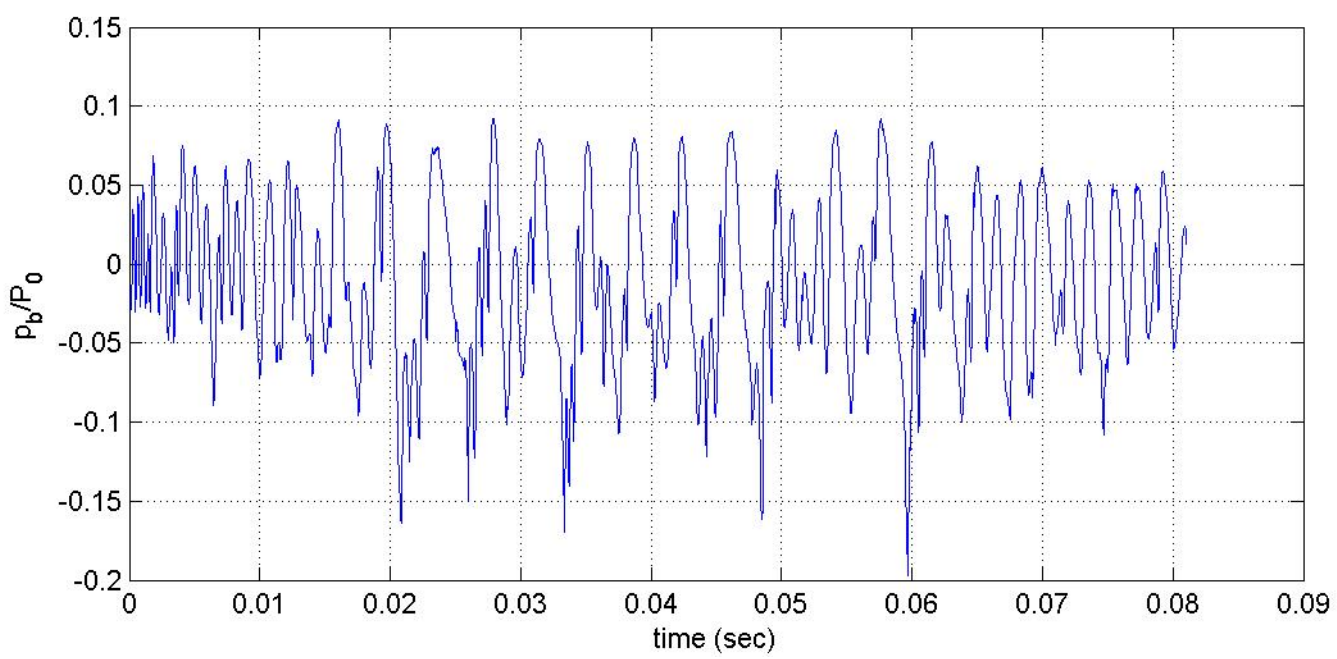

Figure 8. Time trace of the back pressure for $\mathrm{L}=0.35 \mathrm{~m}$.

Since the Large-Structure Model properly predicts most of the essential physics of a free shear layer, one can try to predict the shear layer development in a cavity. Instead of working with solid boundaries and modeling the impingement process on the back wall, a simple feedback was used as follows:

1. The pressure $\mathrm{p}_{\mathrm{b}}(\mathrm{t})$ (later called the back pressure) inside of the shear layer at the distance $\mathrm{x}_{\mathrm{p}}=\mathrm{L}$ was calculated.

2. To model acoustical waves inside the cavity, the back pressure $\mathrm{p}_{\mathrm{b}}$ is delayed by $\tau=L / \mathrm{a}$, where $\mathrm{a}$ is the speed of sound.

3. Emerging vortices are displaced vertically proportional to the delayed back pressure, $\Delta \mathrm{y}_{\mathrm{i}}(\mathrm{t}) \sim \mathrm{p}_{\mathrm{b}}(\mathrm{t}-\tau)$

Obviously, this simplistic approach does not resolve all complex details of the cavity resonance, but one can hope to capture essential physics of the feedback in the shear layer. Calculations were performed for 2000 time steps for three lengths, $\mathrm{L}=0.24,0.35$ and $0.5 \mathrm{~m}$ and both $\operatorname{OPD}(\mathrm{x}, \mathrm{y})$ and $\mathrm{p}_{\mathrm{b}}(\mathrm{t})$ were recorded. An example of back pressure time series for $\mathrm{L}=0.35 \mathrm{~m}$ is presented in Figure 8. With the feedback presence, the shear layer quickly locks itself into one of the Rossiter modes after some transient period (in this case after $0.02 \mathrm{sec}$ ). A close inspection of time series reveals that the feedback bypasses the natural 2-vortex pairing and forces a multiple-vortex merging instead (in this case, 6-8 vortices), creating large structures in the shear layer. Pressure spectra for all cavity lengths are given in Figure 9. First two Rossiter modes predicted by equation (6) are given by black vertical lines on each plot. Natural shear layer case is also presented in Figure 9, upper left plot for comparison. The back pressure spectra for the feedback cases are noticeably different from the natural shear layer case. For each length, pressure signals exhibit strong peaks in the spectra, corresponding to the first and the second (and sometimes the third) Rossiter modes. Experimental values are in a good agreement with the theoretical prediction (6), although even a better agreement was reached with $\beta=0.12$ (not shown here). 

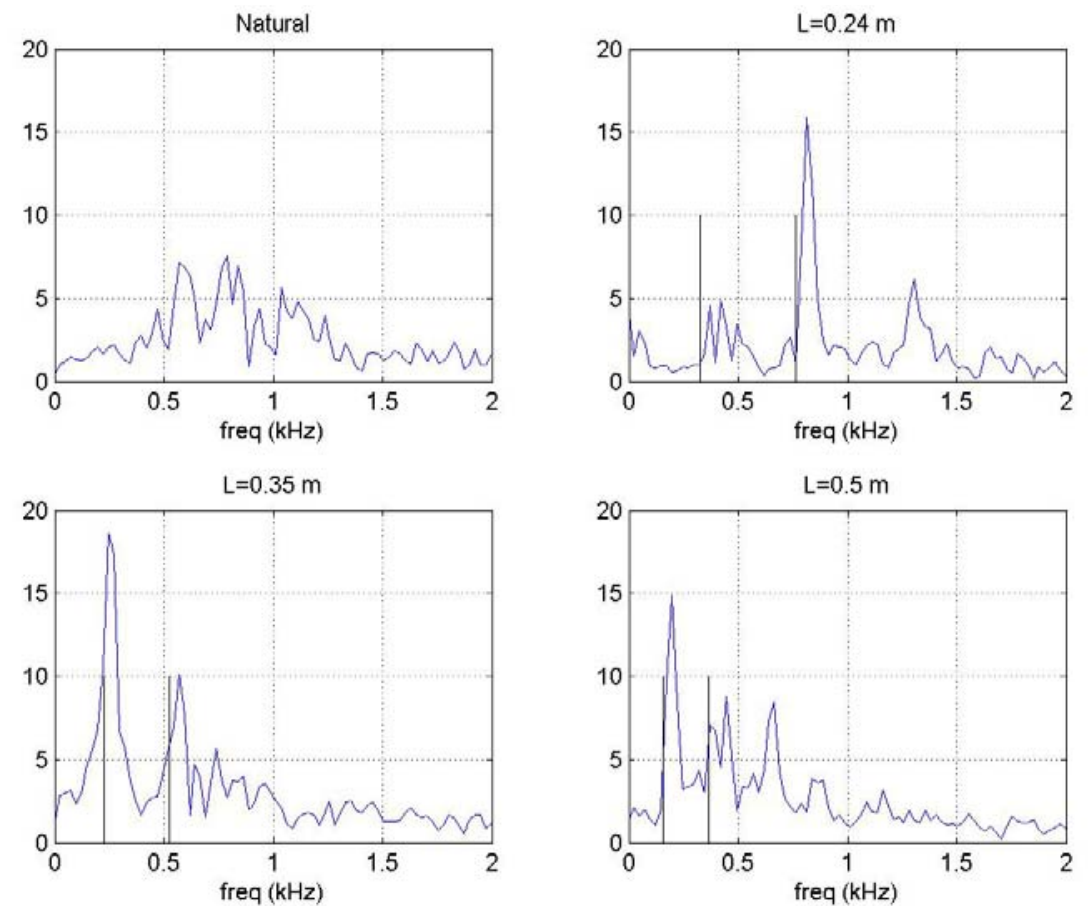

Figure 9. Back pressure spectra for natural shear layer and three cavity lengths.

Corresponding OPD spatial-temporal evolutions are presented in Figure 10. They also show that the presence of the feedback does modify the natural shear layer evolution, presented in Figure 10, upper left plot. Large-scale structures with corresponding pressure drops (blue regions in Figure 10) and consequential changes in OPD are clearly visible. The enhanced feedback forces structures to form earlier and grow faster. The cavity length sets different modes to be dominant in the shear layer. The mode switching can be seen, for instance for the $\mathrm{L}=0.24 \mathrm{~m}$ case (Figure 10, upper right plot) where the second Rossiter mode was dominant up to $0.05 \mathrm{sec}$, followed by the dominance of the first Rossiter mode after 0.05 seconds.
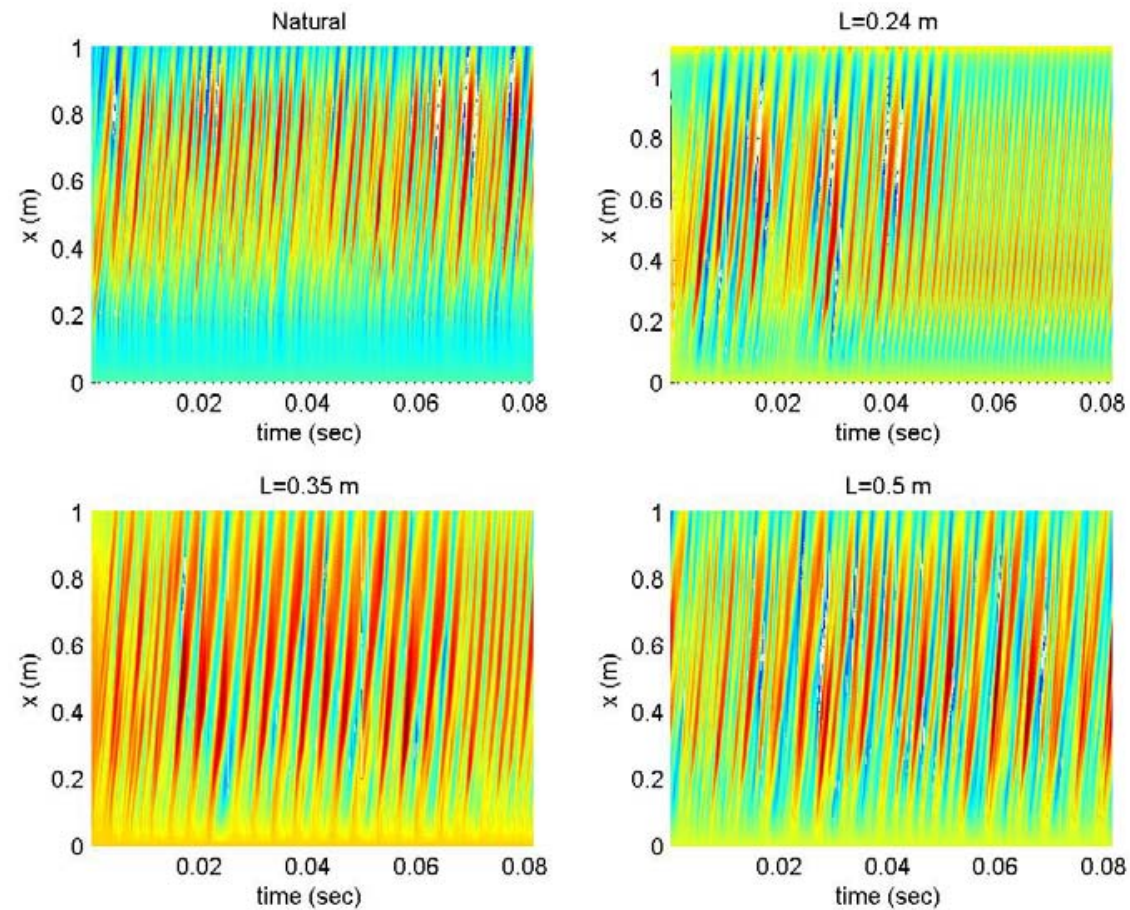

Figure 10. $\mathrm{OPD}(\mathrm{x}, \mathrm{t})$ plots for the natural shear layer, upper left plot, and three cavity lengths. 
Cavity enhances low-frequency, large structures in the shear layer, thus creating quite strong optical distortions on the laser beam when propagating through of the cavity. Streamwise dependence of $\mathrm{OPD}_{\mathrm{rms}}$ for all cases are shown in Figure 11. Indeed, $\mathrm{OPD}_{\mathrm{rms}}$ grow faster in the beginning of the cavity and are higher for all feedback cases, compared with the natural case. Since the simple feedback is imposed at the end of the cavity $\mathrm{x}_{\mathrm{p}}=\mathrm{L}$, it does not effect the shear layer growth downstream of this location. Thus, the shear layer switches back to the natural shear layer evolution beyond $\mathrm{x}_{\mathrm{p}}=\mathrm{L}$ and $\mathrm{OPD}_{\mathrm{rms}}$ grows at a lower rate than upstream of $\mathrm{x}_{\mathrm{p}}=\mathrm{L}$, which is clearly visible in Figure 11.

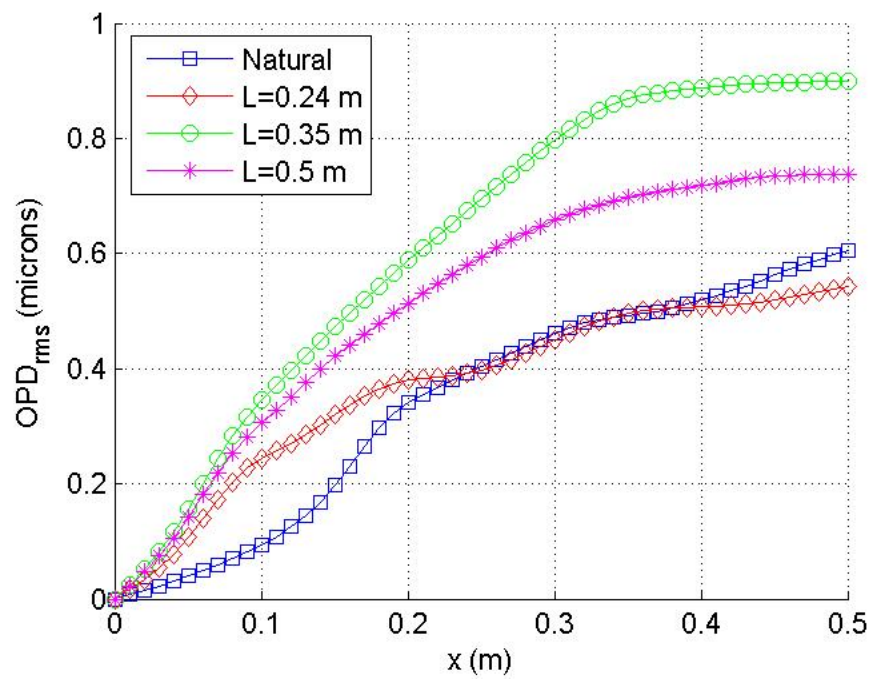

Figure $11 . \mathrm{OPD}_{\mathrm{rms}}$ for natural and cavity shear layers.

\section{Incorporating the LSM code into an aero optical test simulator}

The OPD computations available from the LSM codes are currently being incorporated into an aero optical test simulator (AOTS) code $^{19}$ that was developed to augment aero optical testing and data interpretation. The AOTS code simulates complex aero optical effects by distributing time varying phase screens over a volume in space, each of which represents individual turbulent layers, and then propagating a wavefront through all of the phase screens. Each phase screen is derived by an appropriate turbulence/aero optical model designed to mimic a specific type of turbulence. The LSM code provides all of the information needed to produce phase screens that represent twodimensional, subsonic, compressible shear layers, adding an extremely useful feature to the capability of the AOTS code.

\section{Conclusions}

The simple Large Structure Model (LSM) was developed to quickly estimate optical aberrations caused by the subsonic compressible two-dimensional shear layers. It models each vortical structure as a round vortex with predetermined size and azimuthal velocity profile. Pairing and merging processes are parametrically modeled to further simplify vortical structures' interaction. As vortices are kept round throughout their evolutions, an inner shear layer vortical structure is not resolved. Since only few large vortical structures are present at any moment of time in the computational region, it greatly expedites computational times to solve model's governing equations (2). The model was intensively tested and it was found to correctly predict mean flow quantities and spatial-temporal characteristics of optical distortions caused by a shear layer. Finally, an addition of a simple physical feedback into the model allows to correctly predicting the appearance and evolution of Rossiter modes in rectangular cavities.

The small number of equation in LSM needed to solve to model shear layers makes it a really attractive engineering tool. Although it does not resolve fine details of shear layer vortical structures, it does indeed correctly predicts the essential dynamics of 2-D shear layers, both natural and cavity-based ones, including correct level of optical aberrations. Currently this model is been incorporated into a commercially available optical test simulator to predict far-field patterns and estimate requirements for adaptive optical systems for shear-layer dominant flows. 


\section{Acknowledgments}

The work was partially supported by the U.S. Air Force, Arnold Engineering Development Center under and SBIR program. The authors wish to thank Ron Bishel, Tom Bentley, and Robert Howard of AEDC for support.

\section{References}

\footnotetext{
${ }^{1}$ Thomas, F. O., ”Structure of mixing layers and jets,” Appl. Mech. Rev., Vol. 44, No. 3, 1991, pp. 119-153.
}

${ }^{2}$ Fitzgerald, E.J. and Jumper E.J., “The Optical Distortion Mechanism in a Nearly Incompressible Free Shear Layer,” Journal of Fluid Mechanics, Vol. 512, 2004, pp. 153-189.

${ }^{3}$ Gilbert, K.G., “Overview of Aero-Optics,” Aero-Optical Phenomena, Eds. K.G. Gilbert and L.J. Otten, Vol. 80, Progress in Astronautics and Aeronautics, AIAA, New York, 1982, pp. 1-9.

${ }^{4}$ Jumper, E.J. and E.J. Fitzgerald, "Recent Advances in Aero-Optics,” Progress in Aerospace Sciences, Vol. 37, 2001, pp. 299-339.

${ }^{5}$ Nightingale, A.M., Gordeyev, S., Jumper, E.J., Goodwine, B and Siegenthaler, J.P., "Regularizing Shear Layer for Adaptive Optics Control Applications, “ AIAA Paper 2005-4774, June 2005.

${ }^{6}$ Drazin, P.G. and Reid, W.H., Hydrodynamic Instability, Cambridge University Press, 1981.

${ }^{7}$ Ho, C.M. and Huang, L.S., "Subharmonics and Vortex Merging in Mixing Layers,”, Journal of Fluid Mechanics, Vol. 119, 1982, pp. 443-473.

${ }^{8}$ Leonard, A., “Vortex Methods for Flow Simulation,” J. Comput. Phys., Vol. 37, 1980, pp. 289-335.

${ }^{9}$ Sarpkaya, T., “Computational Methods with Vortices,” J. Fluids Eng., Vol. 111, 1989, pp. 5-52.

${ }^{10}$ Wang, H.Y., Chan, C.K. and Wang, X.L., “ Numerical Investigation Of Mixing Layers Using The Discrete Vortex Method With The Diffusion Velocity Model,”, Numerical Heat Transfer, Part B, Vol. 38, 2000, pp. 223-236.

${ }^{11}$ Siegenthaler, J.P., Gordeyev, S. and Jumper, E.J., "Mapping the Optically-Aberrating Environment in a Partially-Quieted Mach 0.6 Free Shear Layer, “ AIAA Paper 2003-3607, June 2003.

${ }^{12}$ Siegenthaler, J.P., Gordeyev, S. and Jumper, E.J., “Shear Layers and Aperture Effects for Aero-Optics, “ AIAA Paper 2005-4772, June 2005.

${ }^{13}$ Brown, G. L. and Roshko, A., “On Density Effects and Large Structure In Turbulent Mixing Layers,” Journal of Fluid Mechanics, Vol. 64, No. 4, 1974, pp. 775-816.

${ }^{14}$ Rockwell, D. and Naudascher, E., “'Self-sustained oscillations of impinging shear layers,”' Ann. Rev. Fluid Mech., Vol. 11, 1979, p. 67.

${ }^{15}$ Komerath, N. M., Ahuja, K. K. and Chambers, F. W., "Prediction and measurement of flows over cavities-a survey,' AIAA Paper 87-0166, 1987.

${ }^{16}$ Chokani, N.,, “Flow induced oscillations in cavities—a critical survey,’’ AIAA Paper 92-02-159, 1992.

${ }^{17}$ Rossiter, J. E. , "'Wind-tunnel experiments on the flow over rectangular cavities at subsonic and transonic speeds," Aeronautical Research Council Reports and Memo No. 3438, 1964.

${ }^{18}$ Heller, H. H., Holmes, D. G. and Covert, E. E., “'Flow-induced pressure oscillations in shallow cavities,” AFFDL-TR-70104, 1970.

${ }^{19}$ Trolinger, J D. and Rose, W.C., "Technique for Simulating and Evaluating Aero-Optical Effects in Optical Systems,” AIAA Paper 2005-4654, Toronto, Canada, June, 2005. 